\title{
Correction to: First Development, Optimization, and Stability Control of a Pediatric Oral Atenolol Formulation
}

\author{
Mauro Morri, ${ }^{1}$ Patricia Castellano, ${ }^{2,3}$ Darío Leonardi, ${ }^{3,4,5,6}$ and Silvana Vignaduzzo ${ }^{2,3,5,6}$
}

Published online 16 April 2018

\section{Correction to: AAPS PharmSciTech https://doi.org/10.1208/s12249-018-0992-5}

In the "INTRODUCTION" section, Atenolol is defined in parenthesis as (ATN, Fig. 1). The correct definition is (ATN). Fig 1 corresponds to the RESULTS section.

The online version of the original article can be found at https:// doi.org/10.1208/s12249-018-0992-5

\footnotetext{
${ }^{1}$ Planta piloto de Producción de Medicamentos, Facultad de Ciencias Bioquímicas y Farmacéuticas, Universidad Nacional de Rosario, Suipacha 570, 2000, Rosario, Argentina.

2 Área Análisis de Medicamentos, Departamento Química Orgánica, Facultad de Ciencias Bioquímicas y Farmacéuticas, Universidad Nacional de Rosario, Suipacha 570, 2000, Rosario, Argentina. ${ }^{3}$ IQUIR-CONICET, Suipacha 570, 2000, Rosario, Argentina.

${ }^{4}$ Área Técnica Farmacéutica, Departamento Farmacia, Facultad de Ciencias Bioquímicas y Farmacéuticas, Universidad Nacional de Rosario, Suipacha 570, 2000, Rosario, Argentina.

${ }^{5}$ Facultad de Ciencias Bioquímicas y Farmacéuticas, Universidad Nacional de Rosario, Suipacha, 531, 24, 2000, Rosario, Argentina. ${ }^{6}$ To whom correspondence should be addressed. (email:leonardi@iquir-conicet.gov.ar; svignadu@fbioyf.unr.edu.ar)
} 